\title{
Geochemical constraints on core formation in the Earth
}

\author{
John H. Jones \& Michael J. Drake
}

Lunar and Planetary Laboratory, University of Arizona, Tucson, Arizona 85721, USA

New experimental data on the partitioning of siderophile and chalcophile elements among metallic and silicate phases may be used to constrain hypotheses of core formation in the Earth. Three current hypotheses can explain gross features of mantle geochemistry, but none predicts siderophile and chalcophile element abundances to within a factor of two of observed values. Either our understanding of metal-silicate interactions and/or our understanding of the early Earth requires revision.

CORE formation has been described as perhaps the single most important thermal event in the history of the Earth'. This description is certainly true if the core formed by segregation of metal in an initially homogeneous body the size of the present Earth, as the heat liberated in such an event would raise the temperature of the Earth by $\sim 2,000^{\circ} \mathrm{C}$ (ref. 2 ). However, less extreme models in which core formation is concomitant with accretion appear to be more probable ${ }^{3}$. Regardless of whether core formation resulted in catastrophic changes in the Earth, better knowledge of the core-forming event would certainly elucidate important aspects of the histories of the Earth and Moon'.

Geochemists have traditionally divided the chemical elements into four classes: (1) lithophile-those elements which prefer to be combined with oxygen and which typically reside in rocky material; (2) siderophile-elements which prefer to be in the metallic state; (3) chalcophile-elements preferring to be associated with sulphur; and (4) atmophile_-elements so volatile that they are typically concentrated in a planet's atmosphere. Obviously, membership in one of these classes is not invariant, but is modulated by the ambient conditions. For example, as the temperature increases, elements which normally exist as solids or liquids will vaporize and become gases, and, in fact, the classes of lithophile, siderophile and chalcophile are typically subdivided into groups of elements of similar volatility.

In principle, the record of core formation is contained in the siderophile and chalcophile element contents of rocks derived from the Earth's mantle. Certainly these elements are much more depleted in the upper mantle than lithophile elements of similar volatility, implying that they record a depletion event unseen by the lithophile elements-presumably core formation. In practice, these elemental abundances have been attributed to either gross disequilibrium between the core and upper mantle, or mixing of siderophile- and chalcophile-element-rich material into the upper mantle after core formation, or both ${ }^{5,6}$. In this view, little information about core formation may be obtained from mantle siderophile and chalcophile elements because their abundances were established either after core formation ceased or as the results of processes which never approached equilibrium. The evidence cited for disequilibrium between mantle and core during core formation is simply that the concentrations of siderophile and chalcophile elements in mantle-derived rocks are orders of magnitude higher than would be expected if these rocks had ever equilibrated with metal'. Furthermore, many of the siderophile elements in the Earth's mantle exist in chondritic proportions (that is, with elemental abundance ratios characteristic of primitive meteorites (chondrites) which have not undergone chemical processing). Thus, successful models of core formation and for siderophile and chalcophile element abundances in the Earh's upper mantle must be able to explain both high abundances and the apparently unfractionated ratios of some (but not all) element pairs.
Models of core formation are further complicated by our lack of knowledge of the chemical composition of the core. All models of the Earth's core require the presence of a 'light element' to reduce the overall density ${ }^{3}$. If the light element is sulphur, silicon or carbon, then these elements were probably dissolved into the metal (which eventually formed the core) at low pressure as the Earth grew by accretion, and may be relevant to understanding the core-forming process. If the light element is oxygen or a mixture of oxygen and lithophile elements which have followed oxygen into the core, then the light element must have been incorporated at high pressure ${ }^{4}$ and may not have played a role in establishing the siderophile and chalcophile element abundances of the upper mantle.

The behaviours of siderophile trace elements in complex, natural metal-silicate systems are poorly known. This lack of knowledge has led us to determine partition coefficients ( $D s$; for definition, see below) between solid metal, sulphur-bearing metallic liquid and silicate liquid for a suite of siderophile and/or chalcophile elements under controlled laboratory conditions $\left(T=1,250-1,270^{\circ} \mathrm{C} ; \quad P \leqslant 1\right.$ bar $)$ These partition coefficients, together with estimates of the mantle abundances of the same elements, may be used to evaluate three physically plausible but very different hypotheses of core formation. These hypotheses are: (1) inefficient core formation, involving equilibrium between solid and liquid metals and silicates, with a small fraction of solid and liquid metal remaining trapped in the mantle, subsequently to be oxidized ${ }^{7}$; (2) equilibrium between an Fe-S-O metallic liquid and the mantle; and (3) heterogeneous accretion involving a late 'veneer' of oxidized chondritic material which did not segregate into the core $e^{6.9}$. In each of these hypotheses, metal is added to the surface of the growing Earth, and initial interaction between metal and silicate will occur at relatively low pressures. Hence, our partition coefficients, which are obtained at low pressure, may be applicable. Later, we shall return to this point and further discuss the importance of pressure on partitioning equilibria.

A fourth hypothesis, that siderophile and chalcophile element abundances in the Earth's upper mantle were established by partitioning between metal and silicate at very high pressures. perhaps at the core-mantle boundary, will not be explored for three reasons. First, metal-silicate partition coefficients have not been determined at megabar pressures, precluding quantitative evaluation. Second, evidence discussed below suggests that siderophile and chalcophile element concentrations in the mantle were established no later than $3.5 \mathrm{Gyr}$ ago. Thus, material must have been efficiently cycled from the core-mantle boundary to the upper mantle and have been well mixed in $<1$ Gyr. Such effective mixing appears problematical. Third, it seems unlikely that equilibration of siderophile elements between the core and lower mantle would have preserved chondritic ratios of elements such as $\mathrm{Ni}$ and $\mathrm{Co}$, which differ greatly in their siderophile tendencies. 
Table 1 Experimental conditions and results

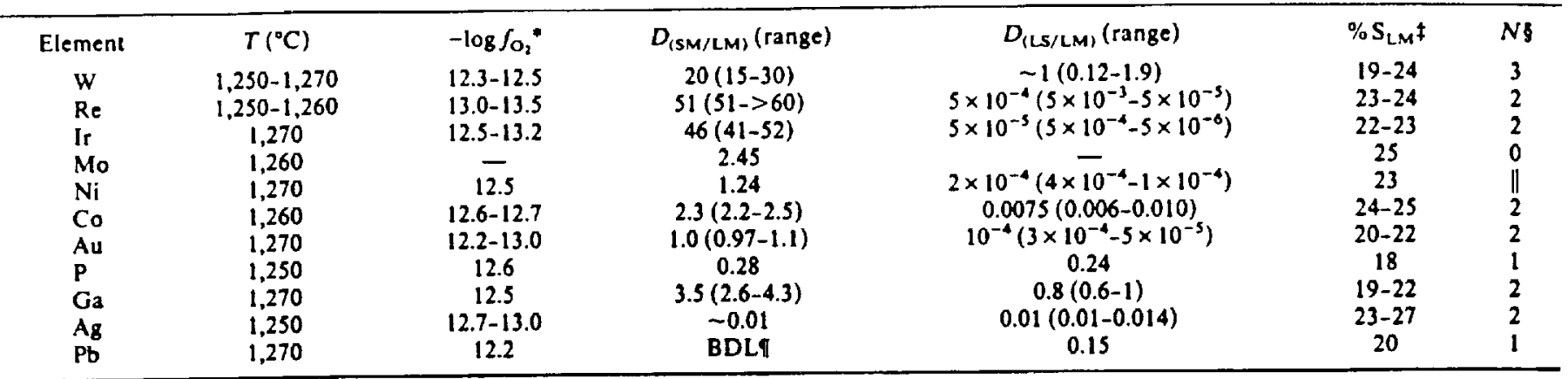

- Calculated from the Fe content of the solid metal and the FeO content of the silicate glass using the iron-wüstite data of ref. 41 .

$¥$ Weight per cent sulphur in liquid metal phase.

$\$$ Number of individual experiments which contained silicate melt.

|| All experiments contain $\mathrm{Ni}$ but only one sample (the most oxidized sample to receive a high-neutron-flux irradiation) contained measurable $\mathrm{Ni}$ in the silicate glass. The range for $D_{\text {(LS/LM) }}$ is that for two analyses of the same glass.

I Below detection limit.

The first three hypotheses will be explored in more detail. First, however, we will discuss constraints obtained from experimental and natural samples which must be satisfied by any successful model of core formation in the Earth.

\section{Constraints}

Partition coefficients. Our experimental partitioning techniques have been described elsewhere ${ }^{10}$ and will not be repeated in detail here. In general, samples containing Fe-Ni metal, natural pyrite $\left(\mathrm{FeS}_{2}\right)$, synthetic basaltic glass and a tracer element are placed in an alumina crucible and sealed in an evacuated silica tube with a separate Fe-metal-silica assemblage designed to maintain the oxygen fugacity of the system $\left(f_{\mathrm{O}_{2}}\right.$, equivalent to the partial pressure of oxygen) near the quartz-fayalite-iron (QFI) buffer (thus ensuring that iron metal is a stable phase). Quenched samples of coexisting solid metal (SM), liquid metal (LM) and liquid silicate (LS) are analysed by electron microprobe. If the tracer concentration in the silicate glass is too low for microprobe analysis, aliquots of glass are separated from metal and analysed by instrumental neutron activation. Table 1 gives experimental conditions and results for $\mathrm{W}, \mathrm{Re}, \mathrm{Ir}, \mathrm{Mo}$, $\mathrm{Ni}, \mathrm{Co}, \mathrm{Au}, \mathrm{P}, \mathrm{Ga}, \mathrm{Ag}$ and $\mathrm{Pb}$-a suite of elements which covers a wide range of nebular condensation temperatures (volatilities) and geochemical behaviour. In this suite $W, R e$ and $I r$ are the most refractory and $\mathrm{Ag}$ and $\mathrm{Pb}$ are the most volatile.

Siderophile abundances in the bulk Earth. The presumed extraction of most of the siderophile and chalcophile elements to the core makes calculation of the bulk-Earth concentrations of these elements difficult or impossible. However, on the basis of lithophile elements, which do not readily combine with $F e$ or $S$, we infer that the bulk Earth is approximately chondritic in composition. We do not mean to imply by this that the Earth is compositionally identical to the group CI chondrites, but that refractory siderophile elements should be present in chondritic relative proportions and that more volatile elements may be depleted to varying degrees. Figure 1 shows the concentrations of siderophile and chalcophile elements in several types of chondritic meteorites. Refractory siderophile elements are usually present in higher concentrations than in group $\mathrm{CI}$, while more volatile elements are typically depleted. Again, although the concentrations of siderophile and chalcophile elements in the bulk Earth are unknown, it is reasonable to require that models of bulk-Earth abundances of these elements be consistent with siderophile and chalcophile element concentration patterns in chondrites.

Siderophile abundances in the upper mantle. During the past decade, a large quantity of high-quality data on mantle samples (such as therzolites) and basalts has become available. Morgan et $a L^{6}$ and Jagoutz et al. " have presented convincing evidence that their lherzolite samples (or at least their most 'fertile' lherzolite samples) have not undergone large amounts of partial melting, and hence are most likely to record primitive mantle abundances, at least for the compatible elements. Thus, when possible, we have used the mantle abundance estimates of refs 6 and 11 . For the incompatibile elements $P, M o$ and $W$, abundances are inferred from the trace-element systematics of basalts (see below). These estimates are listed in Table 2.

Mineral-melt partitioning in basalts. The partitioning of $\mathrm{Ni}$ and Co and between minerals and silicate melts is moderately well known from laboratory experiments, but these elements are the exception rather than the rule. For most of the elements in our suite, solid silicate (SS)/liquid silicate (LS) partition coefficients for element $i$ (' $D_{\mathrm{ss} / L s}$ ) must be evaluated from element correlations in natural basalts. For example, a comparison of data for basalts and ultramafic xenoliths on Re versus Ir and $A u$ versus Ir diagrams from ref. 12 implies that ${ }^{\wedge u} D_{\text {ss/Ls }}$ and ${ }^{\text {Re }} D_{\mathrm{Ss} / L S}$ are of order unity, but that "I $D_{S S / L S}$ is $\sim 50$. (Note in Table 1 that during core formation, trivially small fractions of Au, Re or Ir enter the silicate phase, making the exact value of these particular silicate partition coefficients of little consequence. Also, although the host phase of Ir during silicate partial melting is unknown, the large differences in partitioning behaviour between Au and Ir strongly imply that the host is an oxide or silicate.)

Drake $^{23}$ has used element $i$ versus La diagrams to make geochemical distinctions between terrestrial, lunar and eucritic basalts. Many siderophile and chalcophile elements, when plot-

Table 2 Siderophile and chalcophile element abundances in the upper mantle

\begin{tabular}{ccc}
\hline Element & Concentration $/ \mathrm{Cl}^{*}$ & Ref. \\
W & 0.11 & 15 \\
$\mathrm{Re}$ & 0.0075 & 6 \\
$\mathrm{Ir}$ & 0.0075 & 6 \\
$\mathrm{Mo}$ & 0.064 & 15 \\
$\mathrm{Ni}$ & 0.19 & 11 \\
$\mathrm{Co}$ & 0.21 & 11 \\
$\mathrm{Au}$ & 0.02 & 6 \\
$\mathrm{P}$ & 0.058 & 14 \\
$\mathrm{Ga}$ & 0.3 & 11 \\
$\mathrm{Ag}$ & 0.087 & 6 \\
$\mathrm{~Pb}$ & $0.09 \dagger$ & 42 \\
\hline
\end{tabular}

- Concentrations normalized to CI chondrite ref. 43.

t Mean of total $\mathrm{Pb}$ and unleachable $\mathrm{Pb}$. 


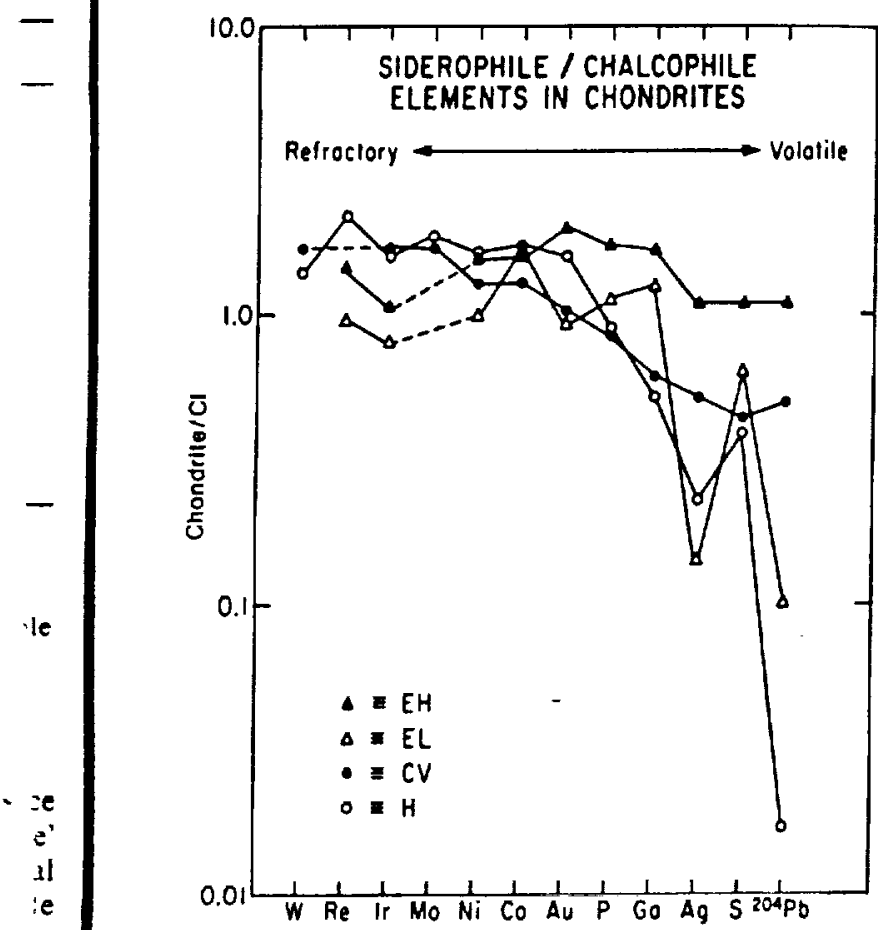

Fig. 1 Siderophile and chalcophile element abundances for four different types of chondritic meteorites $(\Delta, E H ; \triangle, E L ; O, C V$; $\mathrm{O}, \mathrm{H}$ ), normalized to the concentrations of these elements in $\mathrm{CI}$ chondrites. Elements on the left are more refractory and elements on the right are more volatile. Relative to $\mathrm{Cl}$ chondrites, refractory siderophile and chalcophile elements are typically enriched in other chrondrite types and volatile siderophile and chalcophile elements are typically depleted. Chondritic materials provide estimates of the siderophile and chalcophile element abundances that may be expected in the bulk Earth (see text). Data are from the following sources: $\mathrm{E}$, chondrites, refs 45 ( $\mathrm{Re}, \mathrm{Ir}, \mathrm{Ni}, \mathrm{Au}, \mathrm{Ag}$ ), 43 , $46(\mathrm{Co}, \mathrm{Ga}), 43,47(\mathrm{P}), 43,48(\mathrm{~S}), 43,49\left({ }^{204} \mathrm{~Pb}\right) ; \mathrm{CV}$, chondrites, refs 50 (Ir, Co, Ni, Au, Ga), 43, 51 (Re, Ag), 43, 52 (W), 53 (Mo), $43,47(\mathrm{P}), 43,54(\mathrm{~S}), 43,55\left({ }^{204} \mathrm{~Pb}\right)$; H chondrites, refs $43,56(\mathrm{~W}$, $\mathrm{Re}$ Ir, Mo, Ni, Co, Au, P, Ga, S) 43, $57(\mathrm{Ag}), 43,58\left({ }^{204} \mathrm{~Pb}\right)$. When only one reference is given, both the chondrite group analysis and the $\mathrm{CI}$ normalization analysis are reported in that reference. When two references are given, the first is the source of the $\mathrm{Cl}$ normalization.

ted on such a diagram, form linear trends which yield information on the depletion of element $i$ in the mantle (relative to chondritic abundances) and permit the partition coefficient of $i$ between basalt and mantle residuum to be estimated. For example, Ga concentrations in basalts increase by a factor of two, while La concentrations increase by two orders of magnitude in natural terrestrial samples ${ }^{13}$. If we assume that this basalt trend is formed by differing degrees of equilibrium partial melting of similar mantle sources and that ${ }^{L} D_{\mathrm{sS} / L S} \leqslant 10^{-2}$, then ${ }_{G a} D_{\mathrm{SS} / \mathrm{LS}}=0.4$. Correlations of $\mathrm{Pb}$ with La show almost exactly the same variation as the Ga-La diagram, implying that ${ }^{\mathrm{Pb}} D_{\mathrm{SS} / \mathrm{LS}}$ is also $\sim 0.4$. The good (approximately $1: 1$ ) correlation of $P, W$ and Mo with La or another lithophile incompatible element in basalts 14.15 implies that these elements are all very incompatible in silicate systems. Diagrams of phosphorous, W and Mo versus incompatibile elements are also the best means of ascertaining the mantle abundances of these elements ${ }^{15,15}$. Analyses of $\mathbf{A g}$ in terrestrial basalts are very scarce; however, based on the available data ${ }^{16,17}, \mathrm{Ag}$ appears to be weakly incompatible like $\mathrm{Ga}$ and $\mathrm{Pb}$. Consequently, we have adopted a value of ${ }^{{ }^{A}} D_{\mathrm{SS} / \mathrm{LS}}=$ 0.4 , although it could be larger. For example, the MORB (midocean-ridge basalt) data of ref. 17 are consistent with values of ${ }^{{ }^{A}} D_{\mathrm{SS} / \mathrm{LS}}$ between 0.1 and 0.5 , for $5 \%$ equilibrium partial melting.
Summary. Table 3 lists our adopted partition coefficients. As we shall see below, the uncertainty associated with these partition coefficients, while often large, is of the same order as the uncertainties regarding the physical conditions under which core formation occurred. In this sense at least, we feel that zerothorder modelling, using the data of Tables 2 and 3 , is justified. We will now examine hypotheses for core formation in the light of these data.

\section{Hypotheses}

Inefficient core formation. The segregation of the core from the mantle is envisaged as an equilibration between four phases, namely, solid silicate, liquid silicate, solid metal and liquid metal, followed by incomplete separation of metal from silicate. Equilibrium between these four phases is described by three sets of partition coefficients $(D)$ for each element at specified temperature, pressure and oxygen fugacity: $D$ (solid metal/sulphur-bearing metallic liquid), $D$ (liquid silicate/sulphur-bearing

Table 3 Adopted partition coefficients

\begin{tabular}{cccc}
\hline Element & $D_{(\mathrm{LS} / \mathrm{LM})}$ & $D_{(\mathrm{SM} / \mathrm{LM})}$ & $D_{\text {(SS/LS) }}$ \\
$\mathrm{W}$ & 1 & 36 & 0.01 \\
$\mathrm{Re}$ & $5 \times 10^{-4}$ & 83 & 1 \\
$\mathrm{Ir}$ & $5 \times 10^{-5}$ & 83 & 50 \\
$\mathrm{Mo}$ & $8 \times 10^{-4 *}$ & 2.45 & 0.01 \\
$\mathrm{Ni}$ & $2 \times 10^{-4}$ & 1.33 & $10 \dagger$ \\
$\mathrm{Co}$ & $7 \times 10^{-3}$ & 2.3 & $3 \dagger$ \\
$\mathrm{Au}$ & $1 \times 10^{-4}$ & 1.3 & 1 \\
$\mathrm{P}$ & 0.24 & 1.7 & 0.02 \\
$\mathrm{Ga}$ & 0.8 & 6.0 & 0.4 \\
$\mathrm{Ag}_{\mathbf{g}}$ & 0.01 & 0.01 & 0.4 \\
$\mathrm{~Pb}$ & 0.15 & 0 & 0.4 \\
\hline
\end{tabular}

- Calculated from our data and that of ref. 25.

$\dagger \mathrm{Ni}$ and $\mathrm{Co} D_{(\mathrm{ss} / \mathrm{Ls})}$ values estimated from ref. 44 ; all others estimated from natural basalt systematics (see text).

metallic liquid) and $D$ (solid silicate/liquid silicate) where ${ }^{\prime} D_{(\alpha / \theta)}$ is defined to be the weight concentration of element $i$ in phase $\alpha$ divided by the weight concentration of element $i$ in phase $\beta$.

The partitioning behaviour of the èlements in the experimental suite can be generalized in the case of this hypothesis. Mantle concentrations of Au, Ir, Mo, Ni and Re are determined almost solely by the amount of trapped metal phases. Silver is concentrated nearly exclusively in the trapped metallic liquid; $P, W$, $\mathrm{Ga}, \mathrm{Co}$ and $\mathrm{Pb}$ are intermediate in their siderophilic tendencies. Nickel, Co and Ir and compatible $\left(D_{\mathrm{SS} / L S}>1\right)$ in solid silicates (or oxides); W, $\mathrm{P}$ and Mo behave as very incompatible elements $\left(D_{s S / L S} \ll 1\right)$; and other elements are intermediate in their silicate-system compatibility.

Physical conditions of core formation. The fractions of metal and silicate in the Earth are taken to be 0.3 and 0.7 , respectively, the relative masses of the present core and mantle. The proportions of solid metal and liquid metal are determined by assuming that the principal light element in the core is sulphur. Our experiments (see below) were conducted so that our metallic liquids contained $\sim 25 \mathrm{wt} \% \mathrm{~S}$. Thus, if the present core contains $8-12$ wt \% S (ref. 18), use of our experimental data in model calculations requires that the metal assemblage which segregated to form the core was $30-50 \%$ molten at the time of metal-silicate equilibration.

The degree of partial melting of silicate materials during core formation is virtually unknown. If the Earth's accretion and core formation occurred rapidly, it seems inconceivable that high degrees of partial melting could have been avoided'; however, the mantle samples themselves give no evidence for extensive melting early in the Earth's history. Spinel therzolites throughout the world are very similar in composition and 
texture $^{19}$. If therzolites are undepleted in elements that are strongly concentrated in basalts, these lherzolites are described as 'fertile' (that is, able to produce basalt). Large chemical differences are not observed between the fertile spinel therzolites and the fertile garnet lherzolites ${ }^{19}$. Small differences between garnet and spinel therzolites may exist ${ }^{20}$, but the overall chemical similarity between these rock-types implies a mantle which is chemically homogeneous down to at least the depth of the sources of garnet lherzolites $(\sim 150 \mathrm{~km}$; ref. 21$)$. If large degrees of partial melting $(>20 \%)$ occurred in the early Earth, even minor density differences between solid and melt would be expected to lead to phase separation and the formation of a magma ocean ${ }^{22}$. Subsequent crystallization of this hypothetical magma ocean would be unlikely to result in a chemically homogeneous upper mantle. Rather, it would be expected that the formerly molten portion of the mantle would resemble large mafic intrusions with pronounced vertical zoning, as observed in the Stillwater and Skaergaard intrusions and inferred for the Moon ${ }^{23.24}$. Even if such zoning was largely homogenized by subsequent mantle convection, it is surprising that apparently no remnants remain within the continental lithospheres. The uniform chemical composition of mantle lherzolites (the samples from which our mantle siderophile and chalcophile element abundances are mainly derived), coupled with plausible physical theories of core formation which predict continuous core formation as the Earth grows from the mass of Mars to its present mass ${ }^{3}$, lead us to opt for low degrees $(\leqslant 20 \%)$ of silicate partial melting.

The ambient oxygen fugacity during core formation was most likely below the iron-wüstite (IW) oxygen buffer (see later discussion), but is otherwise imprecisely constrained. Thus we allow for isothermal variation in oxygen fugacity in our coreformation model. In practice, this means that we allow $D_{\mathrm{LS} / \mathrm{LM}}$ to change with oxygen fugacity in an ideal manner, as defined by Rammensee ${ }^{23}$. The ' $D_{\mathrm{LS} / \mathrm{LM}}$ values in Table 3 are appropriate for a $\log f_{\mathrm{O}_{2}}$ of -12.75 at $1,250-1,270^{\circ} \mathrm{C}$ (approximately one log unit below the QFI oxygen buffer).

Model calculations. The inefficient core formation hypothesis may be tested mathematically using the formalism outlined below:

(1) After equilibration among the four relevant phases, most of the solid and liquid metal drains away and does not communicate further with the overlying mantle. This process is subject to the constraint of mass balance, so that

$$
\begin{aligned}
{ }^{\prime} C_{\mathrm{Earh}}= & { }^{\prime} C_{\mathrm{SM}} X_{\mathrm{SM}}+{ }^{\prime} C_{\mathrm{LM}} X_{\mathrm{LM}} \\
& +{ }^{\prime} C_{\mathrm{SS}} X_{\mathrm{SS}}+{ }^{\prime} C_{\mathrm{LS}} X_{\mathrm{LS}}
\end{aligned}
$$

where $X$ s are mass fractions, ' $C$ s are mass concentrations of element $i$ and SM, LM, SS and LS refer to solid metal, liquid metal, solid silicate and liquid silicate, respectively. Reiterating the constraints outlined above.

$$
\begin{gathered}
X_{\mathrm{SS}}+X_{\mathrm{LS}}=0.7 \\
p=X_{\mathrm{LS}} /\left(X_{\mathrm{SS}}+X_{\mathrm{LS}}\right) \leqslant 0.2 \\
X_{\mathrm{SM}}+X_{\mathrm{LM}}=0.3 \\
0.3 \leqslant X_{\mathrm{LM}} /\left(X_{\mathrm{LM}}+X_{\mathrm{SM}}\right) \leqslant 0.5
\end{gathered}
$$

where $p$ is the fraction of the silicate portion of the system which is molten. In terms of partition coefficients $(D)$ and degree of partial melting, equation (1) becomes

$$
\begin{aligned}
{ }^{i} C_{\mathrm{Earh}}= & { }^{\prime} C_{\mathrm{LM}}\left[{ }^{i} D_{\mathrm{SM} / \mathrm{LM}}\left(0.3-X_{\mathrm{LM}}\right)+X_{\mathrm{LM}}\right. \\
& +0.7^{\prime} D_{\mathrm{SS} / \mathrm{LM}}(1-p)+0.7^{i} D_{\mathrm{LS} / \mathrm{LM} p]}
\end{aligned}
$$

As partition coefficients have been measured, ' $C_{\text {Earth }} /{ }^{\prime} C_{\mathrm{LM}}$ may be calculated for assumed values of $p$ and $X_{\mathrm{LM}}$. The sensitivity of this calculation to the assumptions of equations (2)-(5) should, in general, be acceptably small. For example, the permissible variation in the total masses of the various reservoirs (solid metal, liquid metal, and so forth) is probably about a factor of two, and the effects of factor-of-two uncertainties translate into two-fold changes in calculated concentrations.

(2) A small amount of trapped solid metal ( $\left.X_{S M}^{\prime}\right)$ and trapped liquid metal ( $X_{L M}^{\prime}$ ) remains behind in the upper mantle. The concentration of element $i$ in the mantle is then given by

$$
{ }^{\prime} C_{\text {manile }}={ }^{i} C_{\mathrm{LM}} X_{\mathrm{LM}}^{\prime}+{ }^{i} C_{\mathrm{SM}} X_{\mathrm{SM}}^{\prime}+{ }^{i} C_{\mathrm{SS}}(1-p)+{ }^{\prime} C_{\mathrm{LS}} p
$$

if $X_{S M}^{\prime}$ and $X_{L M}^{\prime}$ are small $(\sim 0.01)$.

Rearranging as in equation (6), we obtain

$$
\begin{aligned}
{ }^{i} C_{\text {manile }}= & { }^{i} C_{\mathrm{LM}}\left(\mathrm{X}_{\mathrm{LM}}^{\prime}+{ }^{i} D_{\mathrm{SM} / \mathrm{LM}} X_{\mathrm{SM}}^{\prime}\right. \\
& \left.+{ }^{i} D_{\mathrm{SS} / \mathrm{LM}}(1-p)+{ }^{\prime} D_{\mathrm{LS} / \mathrm{LM}} p\right)
\end{aligned}
$$

(3) As ${ }^{\prime} C_{\text {mantle }}$ is known from mantle nodules and basalts, ${ }^{\prime} C_{\mathrm{LM}}$ may be calculated for assumed values of $p, X_{\mathrm{LM}}^{\prime}$ and $X_{\mathrm{SM}}^{\prime}$. If ${ }^{\prime} C_{\mathrm{LM}}$ is known then ' $C_{\text {Earth }}$ can also becalculated from equation (6). The credibility of the chosen values for the model parameters may be evaluated by comparing the calculated ' $C_{\text {Earth }}$ to the concentration of element $i$ in $C I$ chondrites $\left({ }^{\prime} C_{C 1}\right)$. Values of ${ }^{\prime} C_{\text {Eart }}$ for refractory elements which deviate by an order of magnitude from $2-3 \times{ }^{\prime} C_{\mathrm{Cl}}$ are defined as unacceptable, and new values for the model's parameters are chosen.

There are five important adjustable parameters: $X_{\mathrm{LM}}, p, f_{\mathrm{O}_{2}}$ $X_{L M}^{\prime}$ and $X_{S M}^{\prime}$. However, it must be emphasized that it is unknown in detail if the measured partition coefficient values are exactly appropriate. We note that low-pressure ( 1 bar) partition coefficients may be relevant, in that new metal accreting to the Earth is deposited at the surface, and metal-silicate equilibrium is likely to be achieved at relatively shallow depths before the metal largely segregates into the centre of the growing planet. Nevertheless, we should probably be content if any set of model parameters can be found which, assuming an Earth with chondritic relative proportions of refractory elements $(2-3 \times \mathrm{CI})$, will predict mantle abundances of siderophile and chalcophile elements to within a factor of two. Better fits, while mathematically pleasing, may have no physical significance.

Resulrs. Jones and Drake 7 have evaluated inefficient core formation as a means of reconciling the high abundances of siderophile and chalcophile elements in mantle materials with a reducing (that is, low- $f_{\mathrm{O}_{2}}$ ) core-forming event. Early results were encouraging, but increasing the number of constraints on the model (that is, increasing the number of elements to be modelled) has led to progressively poorer model results. Figure 2 shows a 'best fit' calculation of bulk-Earth concentrations for our suite of siderophile and chalcophile elements. The refractory elements $\mathrm{W}$ to Mo have concentrations of $-2 \times \mathrm{CI}$ in the bulk Earth, a reasonable value. The more volatile elemients show various degrees of depletion. Au and $P$ concentrations appear low compared with those of more volatile elements.

Calculated $\mathrm{Co}$ and $\mathrm{Ni}$ abundances are such that the $\mathrm{Co} / \mathrm{Ni}$ ratio in the Earth is within $10 \%$ of chondritic (a requirement of our model), but this agreement is somewhat contrived as $\mathrm{Co}$ and $\mathrm{Ni}$ are not concentrated in the same phase. Nickel is contained mainly in the metallic phases, whereas $\mathrm{Co}$ is retained by the silicates. Maintaining a chondritic $\mathrm{Ni} / \mathrm{Co}$ ratio in the bulk Earth by our model requires trapping large amounts of metallic liquid $(-2.5 \mathrm{wt} \%)$ in the mantle. Although this amount of trapped liquid is not inconsistent with theoretical estimates ${ }^{26}$, the retention of this S-bearing liquid leads to unreasonably high $S$ concentrations $(\sim 6,000$ p.p.m.) in the mantle, in contrast to the observed concentration of $<300$ p.p.m. S (ref. 19)."

Furthermore, because we allow for isothermal variation in oxygen fugacity (and, therefore, in ' $D_{\mathrm{LS} / \mathrm{LM}}$ as well), our best-fit oxygen fugacity is $\sim 1-1.3 \mathrm{log}$ units more oxidizing than the oxygen fugacity recessary for the present mantle $\mathrm{FeO}$ concentra. tions to have been estabished by equilibration with metal at 1 bar. This disagreement is not necessarily a problem, as an oxygen buffer such as QFI will become slightly more oxidizing with pressure (by $-1 \log$ unit per $50 \mathrm{kbar}$ ), but more work is needed to define the relative effects of pressure and oxygen fugacity on siderophile and chalcophile element partitioning. 


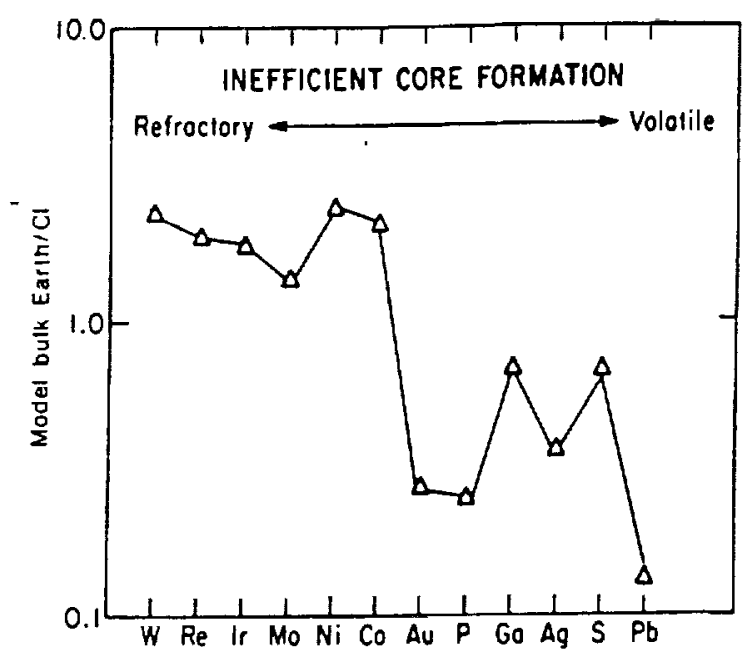

Fig. 2 Calculated bulk-Earth abundances of a suite of 12 siderophile and/or chalcophile elements. Elements are listed in the same order as in Fig. 1. The model of calculation is that of inefficient core formation (see text). The physical conditions of core formation and the amount of trapped metal phases are varied until the calculated refractory siderophile element abundances are 2-3 $\times \mathrm{CI}$ for the bulk Earth and the Ni/Co ratio of the bulk Earth is within $15 \%$ of chondritic. Clearly, geologically reasonable conditions can be chosen so that these two constraints are satisfied. A consequence of the calculation is that volatile elements are found to be depleted in the bulk Earth. A drawback of this type of model is that calculated $S$ abundances for the upper mantle are $>20$ times higher than is actually observed. Best-fit model parameters: trapped metallic liquid (25 wt $\%$ S). $2.5 \%$; trapped metal, $0.04 \%$; degree of silicate partial melting, $10 \% ; \log f_{\mathrm{O}_{2}}=-12.35 ; \mathrm{S}$ concentration in core, $10 \%$.

Thus, even though it is possible to calculate bulk-Earth concentrations of many siderophile and chalcophile elements which are not unreasonable, the consequences of inefficient core formation are not totally attractive. If this model is to be viable, there must be substantial changes in the values of partition coefficients due to an uninvestigated intensive variable (such as pressure). Such variations are certainly not out of the question, especially as the Fe-FeS eutectic composition is known to be pressuresensitive ${ }^{27}$.

Equilibrium between an Fe-S -0 metallic liquid and mantle silicates. This model has been proposed by Brett ${ }^{8}$, and accounts successfully for the upper-mantle abundances of a subset of siderophile and chalcophile elements. The model postulates equilibrium between metal and silicate phases and does not resort to trapping small fractions of metal in the mantle. The main difference between this model and previous equilibrium core-formation models is that no solid metal is present. It recognizes that liquid metal/silicate partition coefficients may be lower than solid metal/silicate partition coefficients (Table 3 ). The redox conditions postulated by Brett ${ }^{8}$ are rather oxidizing, in accordance with the absence of solid metal. This model is mathematically testable using partition coefficients and mass balance constraints in a similar manner to that outlined above. Generalizations concerning element partitioning are also as above.

Alhough at first sight this hypothesis seems successful, there are significant difficulties which appear to invalidate it. For example, the refractory element Mo was calculated by Brett ${ }^{8}$ to be present in the Earth's mantle at $59 \times \mathrm{CI}$ abundance. Although this value is much closer than typical fits obtainable with solid metal/silicate partition coefficients, we consider this to be an unacceprably high value. In addition, although unknown to Brett $^{8}$ at the time, the value of $3.65 \times 10^{-3}$ which he used for the $\mathrm{Ni}$ liquid silicate/liquid metal partition coefficient is very large (compare with our value of $2 \times 10^{-4}$ in Table 3 ) and, in tum, implies an oxygen fugacity too high to be consistent with the low FeO content of the Earth's upper mantle. For example, at $1,250^{\circ} \mathrm{C}$, we predict that the oxygen fugacity required for Brett's $\mathrm{Ni}$ partition coefficient is $\sim 1$ log unit more oxidizing than the $\mathrm{Fe}-\mathrm{FeO}$ (IW) oxygen buffer. Under these conditions, the activity of $\mathrm{Fe}$ in an $\mathrm{Fe}-\mathrm{S}-\mathrm{O}$ metallic liquid will be much less than the activity of $\mathrm{FeO}$ in any coexisting silicates. The very low iron content of the upper mantle $(<10 \mathrm{wt} \%)$ implies that any metallic liquid postulated to have been in equilibrium with the upper mantle (under oxidizing conditions) had an even lower iron activity than the present mantle.

The possibility that core formation in the upper mantle occurred by separation of a metallic liquid with a very low iron activity deserves further exploration. In the Fe-Si system at $1,600^{\circ} \mathrm{C}$, for example, the iron activity at the midpoint of the binary system is $\sim 0.05$ (ref. 28 ). In the Fe-S system at $1,600^{\circ} \mathrm{C}$ the iron activity at the midpoint of the system is $\sim 0.13$ (ref. 29). Non-ideal interactions between iron and non-metals can drastically reduce the activity of iron, even at fairly high iron concentrations in the liquid. Conversely, decreasing the temperature may minimize the non-ideal effects of non-metals. For example, at the eutectics of the Fe-S and Fe-Si systems, where the mole fraction of iron is definitely less than unity, iron metal is stable and the activity of iron in the liquid must also be unity. The complex interaction of intensive variables (pressure, temperature, non-metal concentrations, oxygen fugacity) make quantitative prediction of liquid activities difficult.

Although we cannot rule out highly oxidizing conditions during core formation on physical-chemical grounds, we note that fractionations of the noble refractory siderophile elements are much more probable in this model than in other models of core formation. Because noble siderophile elements appear to be present in chondritic relative abundances in the Earth's mantle, this is an important consideration. In the inefficient core formation model, mantle abundances of the noble refractory siderophile elements are 'buffered' towards chondritic ratios by trapped metal, contrary to the assertion of Morgan ${ }^{30}$. In 'latestage veneer' models, of course, no noble siderophile element fractionations are possible because the material is simply added to the upper mantle without chemical processing.

Figure 3 shows the results of recalculating bulk-Earth abundances for Brett's ${ }^{\$}$ model by extrapolating our internally consistent set of partition coefficients to higher $f_{\mathrm{O}_{2}}$. In this model $X_{\mathrm{LM}}=0.3$, the degree of silicate partial melting is $20 \%$, and $f_{\mathrm{O}}$, is allowed to vary between IW and QFM (quartz-fayalitemagnetite, an oxidizing buffer at which iron metal is unstable). Our inefficient core formation model results from Fig. 2 are shown for comparison. The refractory siderophiles (W, Re, Ir, Mo), rather than being enriched relative to $\mathrm{CI}$, are severely depleted. We conclude that Brett's ${ }^{8}$ equilibrium core formation model is no more viable than our own, although this conclusion is subject to the same caveat as before-that high-pressure partitioning data are virtually non-existent.

Heterogeneous accretion/"chondritic veneer'. The most recent advocates of this type of model are Morgan et al. ${ }^{\circ}$ and Wänke9, who have suggested that material accreted to the Earh became progressively more oxidized as accretion proceeded. Early accretion and core formation was dominated by highly reduced materials, and metal effectively segregated siderophile and chalcophile elements to the centre of the growing planet. By the end of accretion, an influx of more oxidized materials had caused core formation to cease because metal was no longer stable. In this model, moderately siderophile elements such as $\mathrm{Ni}$ and $\mathrm{Co}$ stopped segregating to the core when the Earth's accretion was $80-90 \%$ complete; the noble siderophile elements such as Ir and $A u$ ended their segregation into the core when accretion was $-99 \%$ complete. This model predicts that moderately siderophile elements should be present in the mantle in chondritic ratios, at abundances of $0.1-0.2 \times \mathrm{Cl}$, and that the noble 


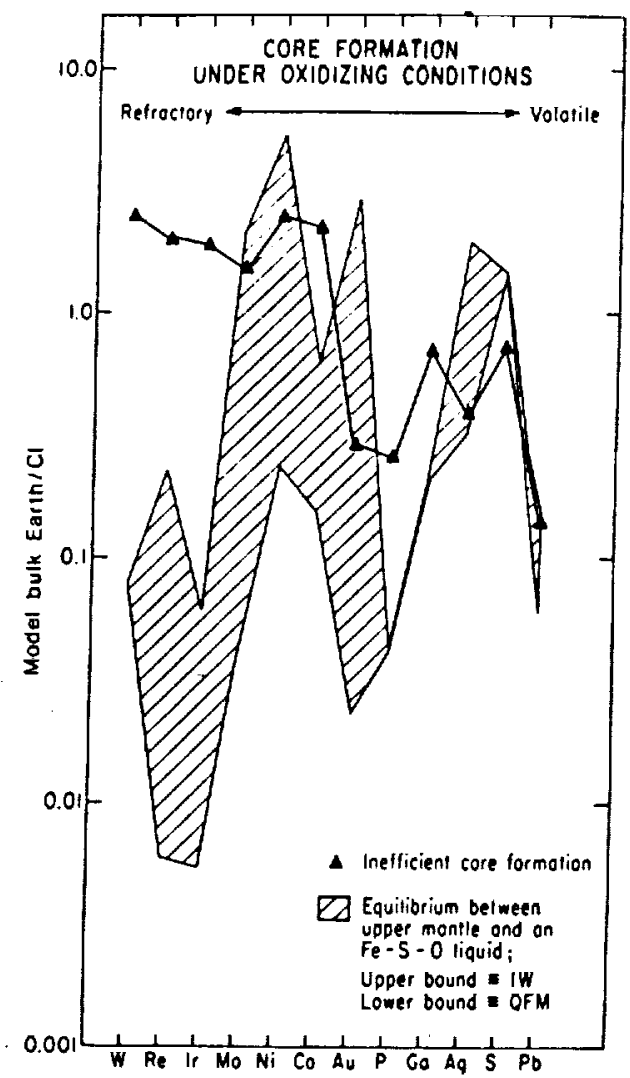

Fig. 3 Calculated bulk-Earth concentrations of siderophile and chalcophile elements assuming equilibrium between an $\mathrm{Fe}-\mathrm{S}-\mathrm{O}$ liquid and a mantle which is $20 \%$ partially molten $\left(T=1,250^{\circ} \mathrm{C}\right)$. The shaded region shows the calculated concentrations for a range of oxygen fugacities, ranging from IW (upper bound) to QFM (lower bound). Bulk-Earth abundaces calculated by the inefficient core formation model are shown for comparison ( $\Delta$ ). The assumption that the core formed in relatively oxidizing conditions, without any trapping of metals by the mantle, leads to the conclusion that the Earth is depleted in refractory siderophile elements, contrary to the expectations based on Fig. 1.

siderophile elements should be present in chondritic ratios at $\sim 0.01 \times C I$ abundances.

This class of hypothesis has gained favour in the past decade, in spite of the requirement that extensive physical mixing of material into the upper mantle must occur after core formation. Impact processes alone seem incapable of such fine-scale mixing (witness the heterogeneity of meteorite-derived siderophile elements in lunar samples), but sustained sub-solidus mantle convection may be adequate, particularly if mantle convection is layered. The mixing process must be capable of homogenizing $\mathrm{Fe}, \mathrm{Co}$ and $\mathrm{Ni}$ at the scale of basalt generation by $3,500 \mathrm{Myr}$ BP and be capable of homogenizing Ir at the hand-sample scale by today ${ }^{7,31}$.

In this regard, it is possible that samples of the upper mantle which have been stored in continental lithospheres for considerable times (without the benefit of sub-solidus convective mixing over geological time) place even tighter constraints on the rate of mixing required to homogenize mantle siderophile elements. Jagoutz et $a l^{32}$ have presented evidence that $2,700 \mathrm{Myr}$-old eclogites have been stored in the lower continental lithosphere for long periods of time (100-2,000 Myr) without participating in mantle mixing processes. More dramatically, Boyd et a ${ }^{21}$ have argued that eclogitic and garnet peridotitic material, transported to the surface by kimberlite eruption, was emplaced in the lower continental lithosphere as long ago as 3,0003,500 Myr. Thus, it is quite possible that the isolation of con-
Table 4 Test of the multi-component accretion model

\begin{tabular}{ccc} 
Element & $\begin{array}{c}\text { Concentration before } \\
\text { second episode of } \\
\text { core formation } \\
\text { (C/CI) }\end{array}$ & $\begin{array}{c}\text { Concentration after } \\
\text { removal of metal* } \\
\text { (C/CI) }\end{array}$ \\
$\mathrm{Ni}$ & 0.2 & 0.2 \\
$\mathrm{Co}$ & 0.2 & 0.2 \\
$\mathrm{P}$ & 0.2 & 0.2 \\
$\mathrm{Mo}$ & 0.2 & 0.064 \\
$\mathrm{Ga}$ & 0.2 & 0.2 \\
$\mathrm{~W}$ & 0.2 & 0.2 \\
\hline
\end{tabular}

- Just enough metal is removed to lower the Mo concentration from 0.2 to $0.064 \times \mathrm{CI}$ (see text).

tinental shield lithospheres from mantle convection and the homogenization of Ir that we observe today in continental lithospheric materials were both complete by 3,500 Myr ago We question whether mantle convection was capable of such rapid, fine-scale mixing.

Another way of making the same observation is to note that Ir contents of lherzolites from beneath old cratons (such as Southern Africa), from beneath younger continental margins (such as the southwestern United States) and from mantle overlying oceanic hot-spots (Hawaii) are essentially identical ${ }^{19}$. The lithospheres beneath southern Africa and the southwestern United States were not obviously isolated from mantle convection at the same time, and the lithosphere beneath Hawaii has presumably never been isolated to the same degree as continental lithospheres. Iridium homogenization was apparently complete by the time of the formation of the earliest continental lithospheres.

Regardless of absolute rates of mantle mixing, the moderately siderophile elements are a sensitive test of the heterogeneous accretion hypothesis. Table 4 shows our model results using the partition coefficients of Table 3 . For simplicity we have quantized accretion and core formation (before the accretion of the last $1 \% \rightarrow$ the hypothesized 'late-stage veneer') into two parts: the first $80 \%$ of material accreted is highly reduced, the last $20 \%$ is more oxidized. This approach permits us to model core formation as two discrete events: the first, which removed all siderophile and chalcophile elements from the early-accreted $80 \%$ of the Earth, and a second event which removed only noble siderophile elements (such as Ir and $\mathrm{Au}$ ) from the late-accreted $20 \%$. A justification of this simple approach is that, because of the large difference in the metal/silicate partition coefficients between $\mathrm{Co}$ and $\mathrm{Ni}$, continuous core formation during the last $20 \%$ of accretion would be unlikely to preserve the approximately chondritic $\mathrm{Co} / \mathrm{Ni}$ ratio of the upper mantle (Table 2). Thus, at the beginning of the second model core-forming event, most (if not all) siderophile and chalcophile elements were present at $\sim 0.2 \times \mathrm{Cl}$ abundances in the upper mantle, as defined by present $\mathrm{Ni}$ and $\mathrm{Co}$ abundances. At this time we allow just enough separation of metal to lower the Mo concentration from $0.2 \times \mathrm{Cl}$ to its present value of $0.064 \times \mathrm{CI}$ (Table 2), and we observe the effect of this separation on the other siderophile elements. As can be seen from Table 4, no moderately siderophile element other than Mo is affected, even though $W$ and $\mathrm{P}$ are clearly depleted relative to $\mathrm{Co}$ and $\mathrm{Ni}$ in the present mantle (Table 2).

The heterogeneous accretion hypothesis results in additional predictions which are inconsistent with observed elemental abundances in the Earth's upper mantle. First, the amount of metal which was extracted from the last $20 \%$ of accreting material must have been trivial, and the high $f_{0}$, requires that that metal must have been $\mathrm{Ni}$-rich (because of the decreased stability of Fe-rich metal). If we assume that as much as $10 \%$ of the total $\mathrm{Ni}$ was removed during the last core-forming event (consistent with the approximately chondritic $\mathrm{Co} / \mathrm{Ni}$ ratio of the upper mantle), then only $0.02 \%$ metal was removed. It would 
seem that a substantially molten upper mantle would be required to separate this minute amount of metal, and, as we have already discussed, there is no evidence for a pervasive terrestrial magma ocean. Second, if the last $20 \%$ of accreted material was sufficiently oxidized to effectively halt core formation, it is expected that this material would have been enriched in volatile elements and oxidized species. If the last dregs of core formation removed only a few hundredths of a per cent of metal, it is not possible for substantial amounts of $S$ to have been taken to the core ( $\mathrm{S}$ is cosmically more abundant than $\mathrm{Ni}$ ). All chondritic materials contain 2-6 wt $\% \mathrm{~S}$. If the material which made up the last $20 \%$ of the Earth's accretion even approached chondritic composition, we would expect that the crust and upper mantle would contain 4,000-12,000 p.p.m. S: not appreciably different from the prediction of the inefficient core formation hypothesis, and in contradiction to observed $S$ abundances of $<300$ p.p.m. in the Earth's upper mantle ${ }^{19}$.

In this context we note that Morgan ${ }^{33}$ has refined his abundance estimates of incompatible noble siderophile elements (for example, $A u$ and $R e$ ) in spinel lherzolites, and finds that the noble element ratios are remarkably chondritic. Obviously, this observation strengthens the arguments for a $1 \%$ chondritic latestage veneer'. Furthermore, Morgan shows that the $S /$ Se ratio of spinel lherzolites is chondritic and advocates that the $S$ and Se abundances of the upper mantle were established by the same 'late-stage veneer' process. The implication of Morgan's work appears to be that, before the last $1 \%$ of accretion, the mantle was sulphur-free. In principle, this may remove some of the difficulties with $\mathbf{S}$ which were discussed above, although it is still unclear whether such low $S$ abundances during the last $20 \%$ of the Earth's inhomogeneous accretion are compatible with the more oxidizing conditions postulated for this stage of accretion?.

We note that the consistency of the siderophile element abundances inferred from basalts, spinel lherzolites and garnet lherzolites implies that the siderophile elements of the upper mantie are likely to be homogeneously distributed to a depth of at least $500 \mathrm{~km}$ - a scale consistent with models of upper mantle convection $^{34}$. If $1 \%$ of the mass of the upper mantle were added by a uniform flux of planetesimals and if this same population were responsible for the production of a $2.5-\mathrm{km}$ megaregolith on the $M_{0 o n^{24}}$, we would expect that the lunar megaregolith would contain $-200 \%$ of chondritic material-a physically impossible value which is about two orders of magnitude higher than the $\sim 1.5 \mathrm{wt} \%$ of meteoritic material measured in lunar soils ${ }^{24}$. The Moon would have to be well-mixed to a depth of $400 \mathrm{~km}$ in order to be consistent with the terrestrial 'late-stage veneer', yet estimates of mantle abundances of noble siderophile elements ${ }^{13}$ show no evidence of such mixing. Of course, it is possible that the Moon formed after the Earth accreted its final $1 \%$ of mass, although available isotopic evidence points to comparable ages for both objects ${ }^{35}$.

Although we find some elements of the heterogeneous accretion/'chondritic veneer' hypothesis attractive, this hypothesis contains as many quantitative difficulties as the inefficient core formation and metallic liquid/silicate equilibrium core formation hypotheses. It is not clear to us which, if any model of core formation should be preferred.

\section{Conclusions}

The preceding discussion emphasizes that none of the current hypotheses of core formation in the Earth survives quantitative scrutiny. For any of the hypotheses to be correct, our present knowledge of the relevant relative partition coefficients must be accurate to perhaps no better than a factor of two. We emphasize that the volume of $\left(P-T-f_{\mathrm{O}_{2}}\right)$ space which has been investigated in our partitioning studies (as well as those of others) is small and should be extended.

This failure of all hypotheses to quantitatively model core formation and siderophile and chalcophile element abundances in the upper mantle is disappointing, in that a detailed picture of the history of the early Earth is important to our understand. ing of many consequential issues, such as the origin of the Moon and the subsequent geological evolution of the Earth. What, if any, statements about core formation may be made with such confidence that they will go unchallenged?

Lack of equilibration between core and upper mantle. All viable models for the explanation of the high abundances of siderophile and chalcophile elements in the mantle implicitly assume that the core and upper mantle have not communicated over geological time. An immediate consequence of this assumption is that either convection in the mantle is layered and the upper and lower mantles communicate only with great difficulty, or the mantle which we sample today was incorporated into the subcontinental lithosphere extremely early. In fact, these two possibilities are not mutually exclusive; in any case the upper mantle and core have not equilibrated since the last addition of siderophile elements to the upper mantle.

Antiquity of siderophile and chalcophile elements in the upper mantle. However the abundances of siderophile and chalcophile elements were established, they are unlikely to have changed over geological time. Evidence from basalts indicates that the $\mathrm{Co}, \mathrm{Fe}$ and $\mathrm{Ni}$ contents of the upper mantle have not changed since 3,500 Myr (ref. 31) and that 'future leads' cannot be explained in terms of continuous core formation ${ }^{36}$. Siderophile and chalcophile element concentrations in the upper mantle were established early in the history of the Earth and are not the result of secondary processes.

The redox state of the early Earth. All models of core formation seem to require that the mantle has become more oxidized over geological time. If the $\mathrm{FeO}$ content of the upper mantle is the result of equilibration with metal, then the consistent $\mathrm{FeO}$ content of $8-10 \mathrm{wt} \%$ in fertile mantle nodules appears to limit the oxidation state of the early Earth to oxygen fugacities less than $-1.5-2.0 \mathrm{log}$ units below the QFI buffer. (For a discussion of the assumptions involved in this calculation see ref. 37.) This estimate should be accurate and is tantalizingly close to the oxygen fugacity of the metal-olivine-pyroxene reaction of ref. 38. If the FeO content of the mantle was not established by reaction with metal but, rather, by later admixture of FeO-rich material, then the calculated oxygen fugacity is an upper limit to that obtaining during core formation. If the $\mathrm{FeO}$ content of the mantle was due primarily to reaction with a metallic liquid, rich in non-metals ${ }^{8}$, the oxygen fugacity was probably still within two log units of IW. In all these cases the mantle was once very reduced and its oxygen fugacity must have changed over geological time to its present oxidized state (that is, from near IW (or lower) to QFM; ref. 39). If the oxygen fugacity calculated at the beginning of this section is accurate, no reactions other than the Fe metal-olivine-pyroxene reaction ${ }^{38}$ need be postulated; if the calculation is only an upper limit, it may be necessary to consider seriously the hypothesis that the Earth is predominantly made of enstatite-chondrite-like material.

Also, we note that the moderately low non-metal concentrations $(8-12 \%)$ inferred for the core (compared with the high concentrations $(-30 \%)$ of $\mathrm{S}$ and $\mathrm{O}$ in the metallic liquid of Brett ${ }^{8}$ ) appear to require that models of core formation which postulate large amounts of $\mathrm{Fe}-\mathrm{S}-\mathrm{O}$-rich liquids cannot apply throughout the core formation process. If non-metal-rich metallic liquids contributed significantly to core formation, then additional metal, containing little or no $S$ or $O$, is required to account for the observed non-metal concentrations in the present core. The easiest means of achieving such a dichotomy is probably through inhomogeneous accretion, in which earlier core-forming events were S-O-poor and later events were S-O-rich. Thus, even models which postulate oxidizing conditions for core formation may require that the oxygen fugacity changed during planetary accretion and core formation, becoming more oxidiz. ing with time.

Future directions. We emphasize again that only a very small 
volume of $\left(P, T, f_{\mathrm{O}_{2}}\right)$-space relevant to core formation has been investigated. Two obvious areas for new experimental explorations are those of high pressure and higher oxygen fugacity. For example, from our work on iron meteorites ${ }^{40}$, we have gained some insight into the possible role of pressure. Many siderophile trace element-non-metal interactions in metallic liquids appear to be explicable in terms of a simple non-metal-avoidance model. Siderophile trace element-sulphur interactions are often $\sim 10$ times stronger than $\mathrm{Fe}-\mathrm{S}$ interactions, which are known to be pressure-sensitive. (Specifically, the composition of the eutectic point in the Fe-FeS binary system becomes more Fe-rich as

1. Brett, R. Reu Grophys Space Phyz 14, 375-383 (1976).

1. Bret, R. Reu Orophys. Space Universicy Press, New Haven, 1952).

2. Urey. H. C. The Planess 1Yale U11-619 (1981).

3. Stevenson, D. Schence 214 the Eerth and Moon (Sprinter, Bertin, 1979).

4. Ringwood, A. E. Ongin of the Eorh and Acte 35, 223-230 (1971).

5. Ringwood, A. E. Geochim cosmochim Acre 2S. Ining. A. J. Proc Lunar planet Sci Conf.

. Morgan, J. W. Wandless

11, $213-233$ (1980).

7. Jones, J. H. Drake. M. J. Eos 62, 1073-1074 11981 , Proce

369-370 (1982); 15, 413-414 (1984): 16, $412-413$ (1985)

8. Breth, R. Geoshim cosmochim Acta 4h, 1183-1188 (1904)

9. Winke, H. Phil Trans $R$ Sac A303, 287-302 (1981).

10. Jones, J. H. \& Drate, M. J. Geochim cormochim Acia 47, 1199-1200 (19).

11. Jagoutz. E. et al Proc. Lunar planet Sri Conf. 10, 2031-20so 19597-AS18 (1983).

12. Chou, C.-L.. Shaw, D. M. \& Crocket, J. H. J. Reophys Res.

13. Drake, M. J. Geochim cosmoxhim Acta 47, 1759-1)67 (1983). 93-100 (1983)

14. Newsom, H. E. Drake, M. J. Geochim cosmochim, Acra $43,93-100$ (1984)

15. Newsom, H. E. A Palme, H. Earth planet Sci Leth A Morgan, J. W. Geochim casmochim

16. Laul, J. C., Keays, R. R., Ga

Acta 34, 329-345 (1972).

17. Hertogen, J., Janssens, M.J. L Palme, H. Geachim cosmochim Acra 4h, 2125-2143 (1980)

17. Hertosen, J., Janssens, M. J. Shampine. D. L. J. geophys. Res, $69,6041-6048$ (1984).

Basaltic Volcanism
Oxford, 1981).

Palme, H. Nickel, K G. Geochin casmochim Acta 49, 2123-2132 (1985)

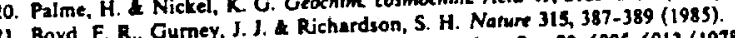

21. Boyd, F. R., Gurney, J. J. \& Ruchardson, S. Heophys Res 23, 6005-6013 (1978)

22. Walker, D., Stolper, E. M. A Hays, J. F. J. Reophys Res

23. Hofmelster, A.M. J. Geophys. Rex. Agllo View (Perzamon, Oxford, 1975)

24. Taylor. S. R. Lunar Science: A Posi-Apollo Ven

25. Rammensee, W. thesis. Unir. Mainz (Iafly Earth: The Interval from Accretion to the Otder

26. Sievenson, D. J. in Workshop on the Early Earth: The (LPI tech. Rep. 85-01, Lunar and Archean (eds Burte, $K$ Ashwal,

Planetary Institute, Houston, 1985).

27. Usselman, T. M. Am. J. Sai 275, 278-290 (1975).

28. Hultgren, R. Pramod, D. D., Hawkins, D. T., Gleiser, M. 2 Kelleys, R. $871-883$ (Ameriean Society of Metals,

of the Thermadynamic

Metals Park, 1973).

29. Sharma, R. C. Chang. Y. A. Merall Trons

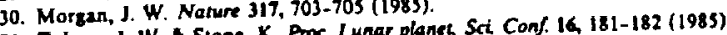

pressure increases ${ }^{27}$.) Extrapolating from the $\mathrm{Fe}-\mathrm{S}$ system, it is possible that siderophile element-non-metal interactions will be possible thations of pressure. Quantitatively successful models of core formation may require accurate and detailed assessments of the $(P, T)$ state of the early Earth.

This work was supported by NASA grant NAG 9-39. We thank $H$. Doane and the Reactor Facility of the University of Arizona for assistance; D. Hill and W. V. Boynton for help with neutron activation analyses; D. Stevenson and F. Richter for discussions; and R. Brett, D. J. Malvin, J. W. Morgan and H. E. Newsom for helpful reviews.

32. Jagoutz, E., Dawson, J. B., Hoemea, S., Spettel, B. \& Winke, H. in Wortuhop on the Earty Earth: The Interval from Aceresion to the Older Anchaen (edi Burke, K. Ashwal. LD.) 40-41 (LPI tech. Rep. 85-01, Lunat and Planetary Institute. Houston, 1985).

33. Morsen, J. W. J. geophyse Res (in the press).

33. Roryer. F. M. Earth planet Sci Lett. 73, 350-360 (1985).

35. Ruing

(Lunar and Planetary Institute, Houston, in the press). 36. Newsom, (1985).

37. Newsom, H. E. J. seophys Rea eA, C613-C617 (1985)

38. Larimer, J. W. Geochim cosmochim Acta 32, 1189-1209 (1968).

38. Lrimer, J. W. Geochin Eerth planet Sci IS, (1985).

19. Arculus, J. A. Rre Eart planel Se. Jechim casmochim Acte (in the press)

40. Malvin, D. Jones,

41. Meyen, J. \& Eugster. H. P. Conre Mci Lett 20, 54-66 (1973).

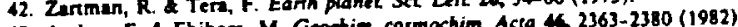

43. Anders, E. \& Ebihara, M. Geochim cosmochim, Acro (1978)

4. Irving. A. J. Geochim caumochim. Acra 12, 743-770 (1978). mochim Acra 47, 2241-2255 (1983).

6. messon, J. T. Geochim cosmochim Acta 4C 597-608 (1982).

17. Yon Michaelis, H. Willis, J. P. Ertant, A. J. \& Ahrens, L H. Earth planet Sci Leth 5 , $383-386$ (1969).

48. Moore, C. B. in Handbook of Elemental Abund (Gordon and Breach, New York, 1971)

(Gordo and Brech,

49. Manhes, G. A Nlegre, C. J. Meteonitice 13, 543-548 (1978).

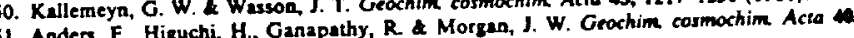
1131-1139 (1976)

52. Morgar J. 224 789-791 (1969).

52. Mortan, J. We

54. Mason, B. Space Sci Rev 1,621-646 (1963).

55. Tatsumoto, (1976).

66. Mason. B. Handbook of Elemental Abundances in Meteories, 35, (Gondon and Breach New York, 1971)

57. Laul, C. Ganapathy, R. Anders, E. \& Morgan, J. W. Geachin cosmachin Acta 37, 329.357 (1973).

58. Tatsumoto, M. Knight, R. J. \& Nlègre, C. J. Science 16h 1279-1283 (1973).

\title{
Binding of a specific ligand inhibits import of a purified precursor protein into mitochondria

\author{
Martin Eilers \& Gottfried Schatz
}

\author{
Biocenter, University of Basel, CH-4056 Basel, Switzerland
}

Methotrexate, a folate antagonist, blocks import into mitochondria of mouse dihydrofolate reductase fused to a mitochondrial presequence. Methotrexate does not mask the presequence, but stabilizes the dihydrofolate reduclase moiety. It does not inhibit import of the authentic precursor from which the presequence is derived. This suggests that dihydrofolate reductase must at least partly unfold in order 10 be transported across mitochondrial membranes.

TRANSLOCATION of proteins across biological membranes is a key step in the intracellular sorting and secretion of proteins. Although much is known about protein movement across various membrane systems ${ }^{1}$, it is unclear how hydrophilic, charged proteins translocate through the hydrophobic core of a phos. pholipid bilayer. In particular, it remains unanswered whether a protein must unfold during the transport process ${ }^{2}$ or whether it can translocate in a folded state ${ }^{3}$, perhaps through transient non-bilayer domains in the target membrane'. We have approached this question by fusing the presequence of an im- 